\title{
Investigation of Flexible Textile Antennas and AMC Reflectors
}

\author{
M. Mantash, A.-C. Tarot, S. Collardey, and K. Mahdjoubi \\ (Institute of Electronics and Telecommunications of Rennes) IETR, University of Rennes 1, Campus de Beaulieu, \\ bat. 11D, 35042 Rennes Cedex, France \\ Correspondence should be addressed to A.-C. Tarot, anne-claude.tarot@univ-rennes1.fr
}

Received 15 December 2011; Revised 15 February 2012; Accepted 17 February 2012

Academic Editor: Hendrik Rogier

Copyright (c) 2012 M. Mantash et al. This is an open access article distributed under the Creative Commons Attribution License, which permits unrestricted use, distribution, and reproduction in any medium, provided the original work is properly cited.

In this paper, two different methods for fabric characterization are presented: a single frequency method and a broadband method. Felt and denim fabrics are characterized, and patch antennas are designed using these substrates to test both methods. Prototypes of the antennas on felt and denim are manufactured using conductive textile (called electrotextile) aiming to obtain fully flexible antennas. The prototypes are characterized in anechoic chamber to be compared and obtain conclusions related to the characterization methods. A new dual-band hexagonal AMC reflector combinable with antennas is also proposed to improve their performance and reduce the backward radiation to the human body. A novel broadband CPW-fed monopole antenna is designed to be combined with the AMC. The resulted prototype is characterized and compared with the performance of the CPW-fed antenna alone.

\section{Introduction}

In recent years, flexible and textile antennas have taken a lot of attention due to their application in wearable systems. A review of wearable textile antennas is presented in [1], whereas, in [2], a GPS textile antenna is tested in realwork conditions. A Bluetooth textile antenna under bending conditions is presented in [3] and has also been combined with artificial magnetic conductors (AMCs [4-7]) aiming to improve their radiation properties [8], reduce the backward scattered radiation to the body $[9,10]$, or cover dual-band applications [9].

Nowadays, research efforts focus on both the design of new antennas and the development of novel textiles to be used as antenna substrates. Wearable antennas need to be integrated within everyday clothing, be low profile, and be hidden as much as possible. There are also other challenging works to maintain the critical antenna parameters at an acceptable level in all "normal" operation condition and environment. For example, stress (bending) can affect the axial ratio, operating frequency, and efficiency; humidity can affect the conductive textiles, by rusting them and changing their conductivity, as well as the dielectric textiles by changing their relative dielectric permittivity [11].

Bearing in mind all these facts, the selection of a proper conductive textile and a substrate for the antenna design are key points. The electrical parameters of garments are generally not available in literature. Therefore, the first step to accomplish is the characterization of the fabric in terms of dielectric permittivity and loss tangent.

In this paper, two methods for fabric characterization are presented. Two well-known fabrics, felt and denim, are characterized to test the methods, and prototypes of patch antennas on these fabrics are manufactured using electrotextiles as conductive parts. Then, these manufactured prototypes are characterized through return loss and radiation pattern measurements in order to obtain some conclusions regarding the characterization methods. In addition, the design of a novel hexagonal dual-band textile AMC to be combined with antennas in order to improve their performance and protect the human body from radiation is presented. A broadband CPW-fed broadband antenna on felt is proposed to be combined with the dual-band AMC. Finally, a prototype of CPW-fed monopole antenna over AMC using felt is manufactured and characterized. It shows the enhancement of the antenna performance when combined with AMC.

\section{Methods of Fabric Characterization}

In order to perform the electrical characterization (dielectric constant $\varepsilon_{r}$ and loss tangent $\tan \delta$ ) of the fabrics, two 


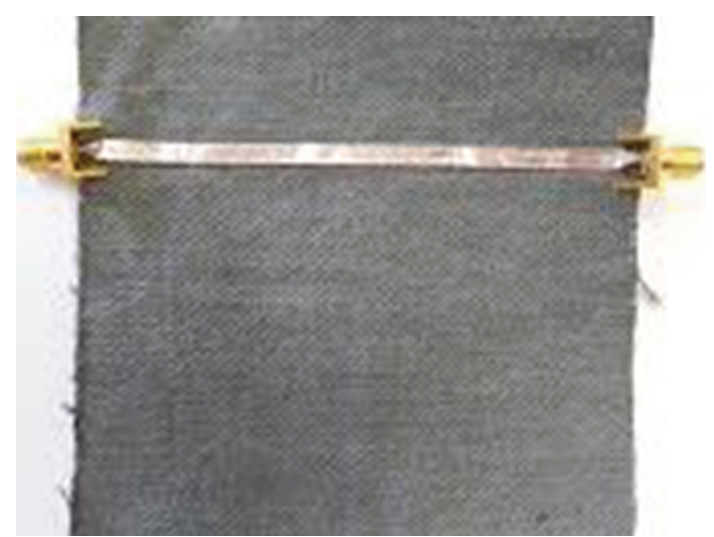

(a)

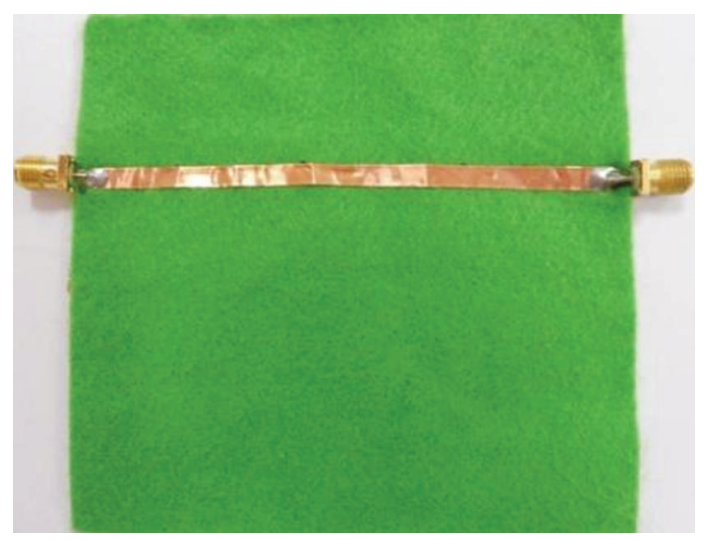

(c)

FIGURE 1: Fabric characterization setup: microstrip line ((a) and (c)) for calibration and open stub ((b) and (d)) for permittivity measurement using denim (up) and felt (down).

different methods were used: the method of stub resonator [12] which is valid for a single frequency and a broadband method based on a stripline cell [13]. Both methods are applied to denim and felt fabrics, and their results will be compared.

2.1. Single Frequency Method. The procedure used in the method of stub resonator [12] to perform the electrical characterization (dielectric constant $\varepsilon_{r}$ and loss tangent $\tan \delta$ ) of the fabric is as follows: first, the set-up calibration is carried out by measuring the $S_{21}$ parameter for a microstrip line (see Figures $1(\mathrm{a})$ and $1(\mathrm{c})$ ) on the fabric. Then, the $S_{21}$ parameter for $\lambda / 4$ stub (see Figures $1(\mathrm{~b})$ and $1(\mathrm{~d})$ ) is measured. After this step, simulation of the $S_{21}$ parameter for the $\lambda / 4$ stub is carried out using HFSS software [14]. The dielectric constant $\varepsilon_{r}$ is then adjusted in simulations in order to match the simulated curve of the $S_{21}$ parameter to the measured one.

This method of characterization is applied to two commonly used fabrics: denim and felt. Cupper tape is used for the metallic parts (ground plane and lines). The line width and stub length are, respectively, $3.6 \mathrm{~mm}$ and $25 \mathrm{~mm}$ for denim, and $4 \mathrm{~mm}$ and $26.5 \mathrm{~mm}$ for felt. For denim, the best match for the resonant frequency is obtained using $\varepsilon_{r}=$

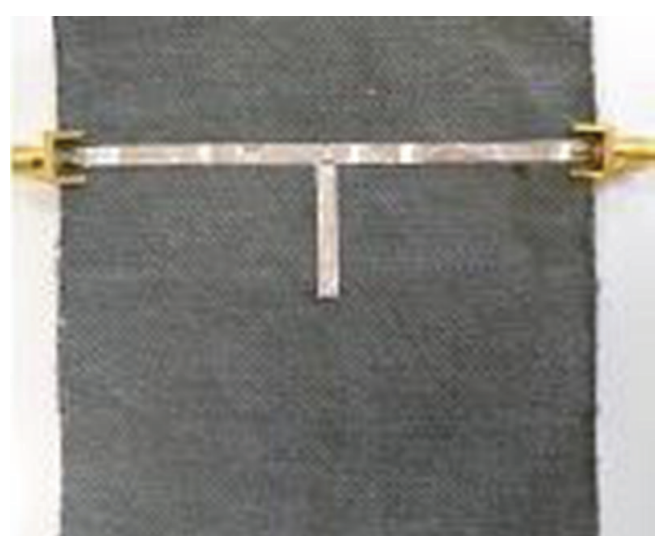

(b)

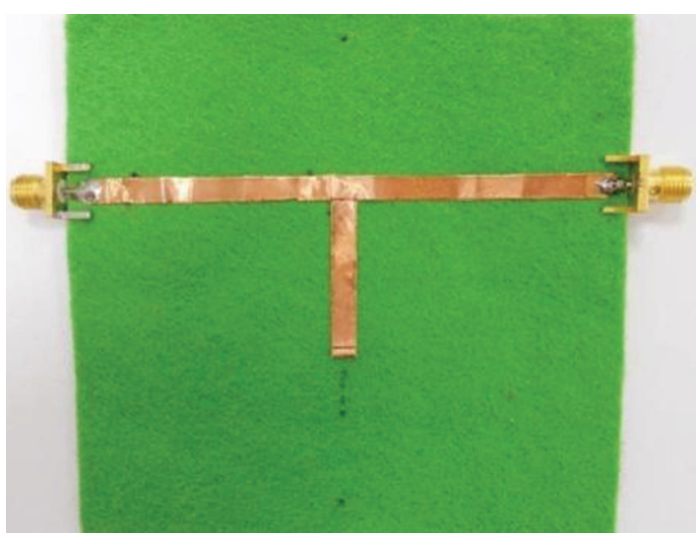

(d)

1.6 (Figure 2). Concerning the loss tangent $(\tan \delta)$, we obtained 0.05 . The estimated values for felt are, respectively, $\varepsilon_{r}=1.22$, and $\tan \delta=0.016$. These estimated values will be used for antennas simulations.

Compared to other characterization methods [13], the advantages of this one are simplicity, rapidity, no need for specific equipment, and, finally, applicability to all types of fabrics.

This method is well suited to textile whose characteristics are not highly variable with frequency (i.e., the textile that is not dispersive). If the textiles are dispersive, that is permittivity varies versus frequency, a broadband method is preferable [13]. However, it needs some specific setup and software.

2.2. Broadband Method. Suitable for measuring the complex this method is based on a stripline topology (see Figure 3). The samples come in the form of rectangular plates or thin films deposited on a dielectric support.

The electromagnetic analysis of the cell is based on a quasistatic approach. This approach represents the crosssection of the stripline containing the tested sample by an equivalent cross-section consisting of a homogeneous permittivity and permeability of isotropic solid materials, 


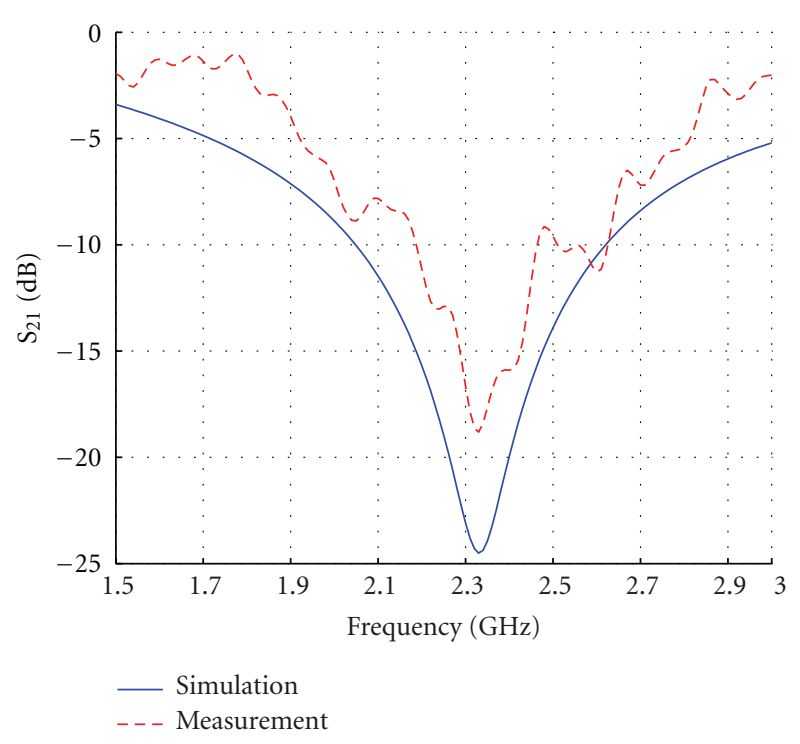

(a)

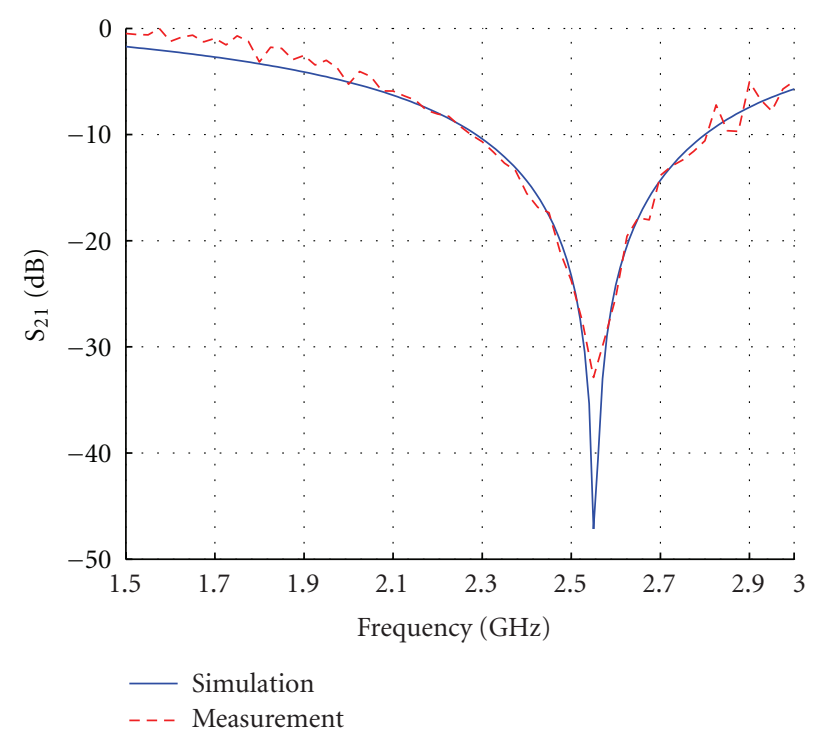

(b)

Figure 2: Simulation and measurement of $S_{21}$ parameter for the open stub on denim (a) and on felt (b).

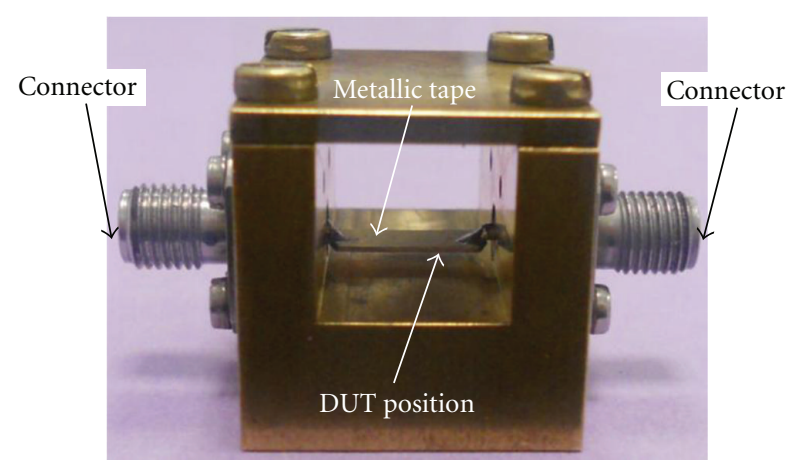

FIGURE 3: Stripline used for measurements.

medium of effective permittivity and permeability $\varepsilon_{\text {eff }}$ and $\mu_{\text {eff. }}$ The measurement parameters $S_{i j}$ are traced back to the actual parameters of the material. The frequency range is typically $300 \mathrm{kHz}-8 \mathrm{GHz}$. The accuracy of the results is high, with a relative error less than 5\% [15]. This method has been used to characterize denim and felt samples to compare the obtained results with those of the single frequency method.

According to this method applied between $1 \mathrm{GHz}$ and $6 \mathrm{GHz}$, the measured values for relative dielectric constant $\varepsilon_{r}$ vary from 1.215 to 1.225 for felt (Figure 4) and from 1.6 to 1.65 for denim (Figure 5). Regarding the loss tangent $\tan \delta$, the values are 0.016 for felt and 0.05 for denim and relative magnetic permeability $\mu_{r}=1$ for both felt and denim fabrics.

Comparing the obtained results using the two methods, it can be observed that both of them estimate the same values for felt, but there are slight differences for denim.

\section{Electrotextiles}

The manufacturing of antennas integrable into clothing has become possible through the use of electrotextile, which

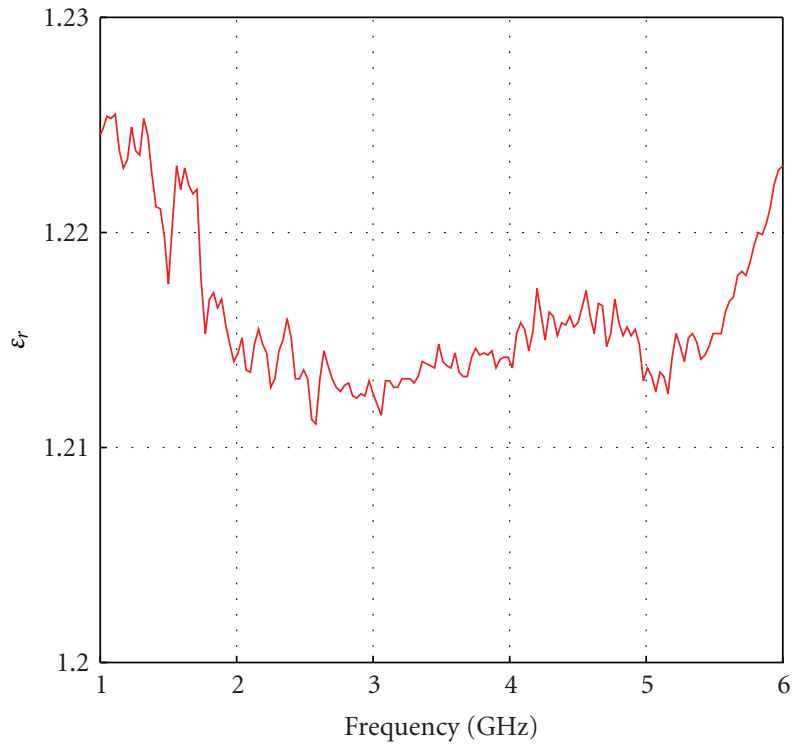

Figure 4: Measured permittivity versus frequency for felt.

are conductive fabrics [16] obtained by interleaving normal fabrics with conductive metal/polymer threads. The selection of an appropriate electrotextile is an important step in the design of a textile antenna. In order to ensure the optimum characteristics for a definite application, the electrotextile for textile antennas should exhibit the following properties: low electrical resistance to minimize losses, flexible to be able to be deformed when worn, inelastic and tear resistant to preserve its electrical properties, and light weight to be comfortable.

Bearing in mind all these facts, two different electrotextiles have been selected in this paper: "Pure Copper Taffeta Fabric" and "Shieldit Super." Properties of such 


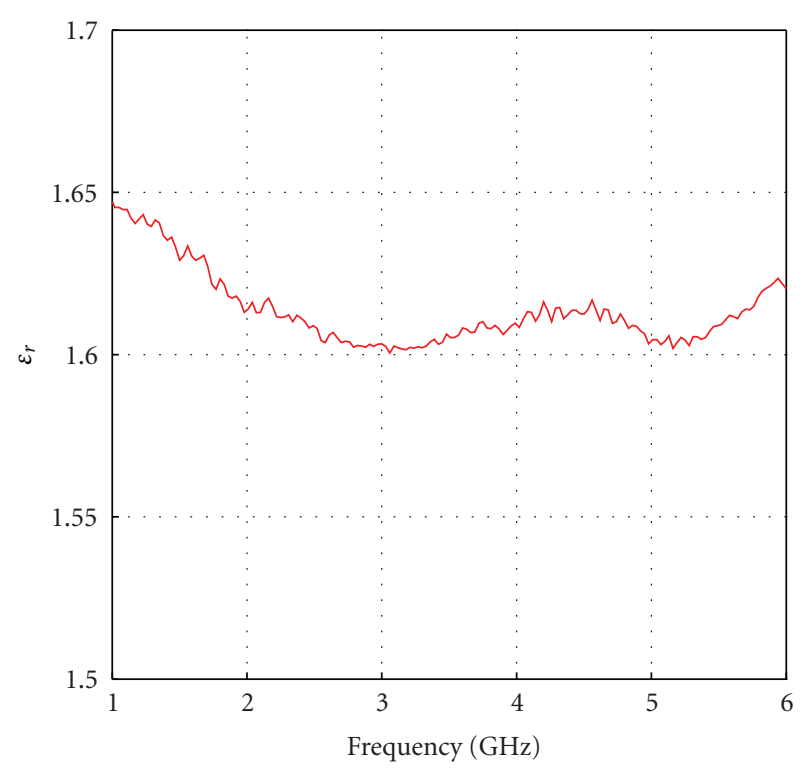

FIGURE 5: Measured permittivity versus frequency for denim.

electrotextiles, provided by supplier are given in Table 1 . Photographs taken through a microscope are presented in Figure 6. Moreover, the resistivity of both electrotextiles has been measured in our laboratory IETR, using the "4-probe setup."

"Pure Copper Taffeta Fabric" is made using pure copper and has the advantages of being smooth, flexible, light weight, easy to be cut and sewn.

An additional adhesive layer is unfortunately needed to fix the electrotextile to the fabric, thus increasing the losses.

"Shieldit Super" is made of polyester substrate conductive nickel and copper plated then backed with nonconductive hot melt adhesive. It has the advantages of excellent shielding and low corrosion, it is also easy to be cut and sewn, and, additionally, its adhesiveness is activated at $130^{\circ} \mathrm{C}$ $=266^{\circ} \mathrm{F}$. It can be ironed on to cotton or another fabric. The use of polyester substrate instead of nylon (as in another electrotextile) makes it better for moisture management as it is more hydrophobic and (more difficult it to become wet) which allow to better conserve its electromagnetic properties. For these reasons, Shieldit Super will be used in next section. As a drawback, it can cause skin irritation because of containing nickel, but this is not a major concern in the design presented here as an additional fabric layer can be used to isolate the electrotextile from the body.

\section{Antennas on Textile}

Two patch antennas at $2.45 \mathrm{GHz}$ using denim and felt fabrics as substrate are designed and characterized. Both use Shieldit Super as electrotextile.

Figure 7 shows the manufactured prototypes. The patch dimensions as well as the return loss and realized gain are given in Figure 8 and Figure 9 for felt and denim fabrics.
Figure 10 shows the setup for measuring the radiation pattern of the prototypes using Satimo stargate SG 32.

There is a good agreement between the measurement and the simulation results both for felt and denim substrates. A slight frequency shift between the simulation and experimental results is observed both for $S_{11}$ and gain parameters. It can be attributed to the manufacturing tolerances.

Regarding the radiation patterns (see Figure 11), one observes a good agreement between the measured and simulated curves for the principal polarization and a satisfactory one for the cross polarization.

\section{Artificial Magnetic Conductor (AMC) on Textile}

The textile antennas usable in wearable applications can be combined with artificial magnetic conductors (AMCs) with the aim of improving the antenna's radiation properties and reducing their backward radiation to the human body [4-7]. These performances are achieved due to the AMC in-phase reflection property. Here, a dual-band AMC, resonating at $2.4 \mathrm{GHz}$ and $5.5 \mathrm{GHz}$, is designed to be combined with the dual-band CPW-fed antenna on felt.

The resonance frequency and the bandwidth of an AMC reflectors are determined by the unit-cell geometry, the dielectric permittivity, and the substrate thickness. Thus, for a given dielectric substrate, the unit-cell design plays an important role to obtain dual-band behavior and to ensure sufficient bandwidth at each subband.

Figure 12 shows the geometry of the proposed AMC for dual-band operation. The unit-cell consists of an inner hexagonal patch surrounded by a concentric hexagonal loop. The hexagonal shape offers a rotational symmetry for the wave propagation and the best filling factor which allows the maximum metallic size and consequently the best bandwidth results. Dual-band behavior is due to the fact that the central patch and the surrounding ring have two different resonance frequencies.

Shieldit electrotextile is used for the metallic elements (hexagon unit-cells and the ground plane under the dielectric substrate) and felt fabric with $h=4 \mathrm{~mm}$ (thickness), $\varepsilon_{r}=$ 1.22 , and loss tangent $\tan \delta \cong 0.016$.

To find the frequency band in which the periodic structure exhibits AMC behavior, we have studied by simulation its reflection coefficient for a uniform plane wave, using commercial software HFSS V.13 of Ansoft [14].

A single cell of the structure has been investigated with periodic boundary conditions (PBCs) on its sides, modeling an infinite structure. Normal incidence plane waves are launched to illuminate the periodic structure using a waveport placed a half-wavelength above it. Following [4], the reflection coefficient phase on the AMC is obtained. The phase reference plane is taken on the periodic surface.

Simulation has shown that the inner hexagon mainly determines the higher resonant frequency. Increasing the elements size, Hex_out and Hex_in, lowers the resonant frequency, while increasing the gap $2 \mathrm{dL}$ between unit cells raises the lower frequency. Hex_cut can be used to control the 


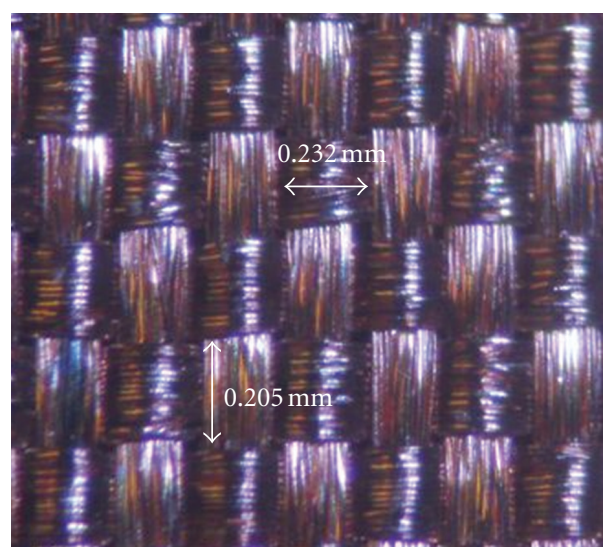

(a)

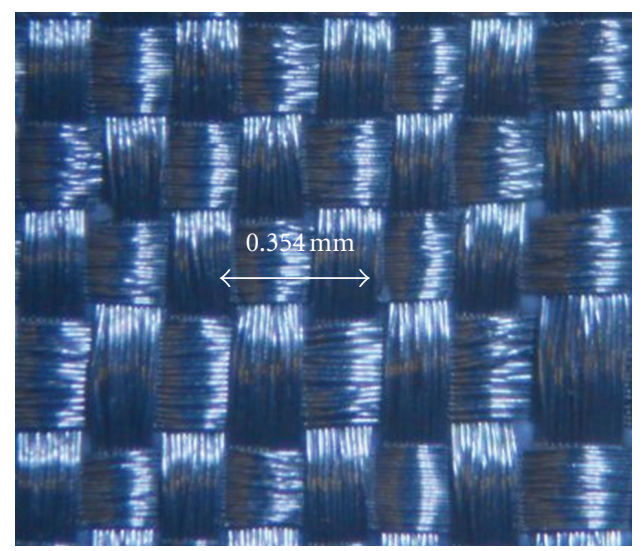

(b)

Figure 6: Microphotographs of (a) Pure Cooper Taffeta Fabric. (b) Schieldit Super.

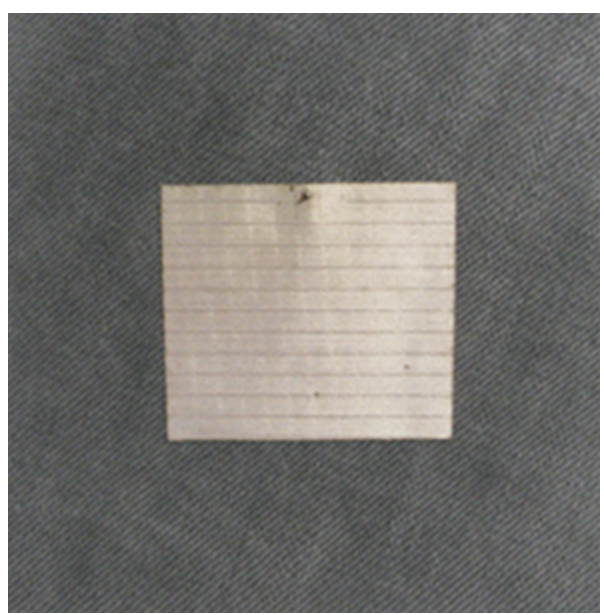

(a)

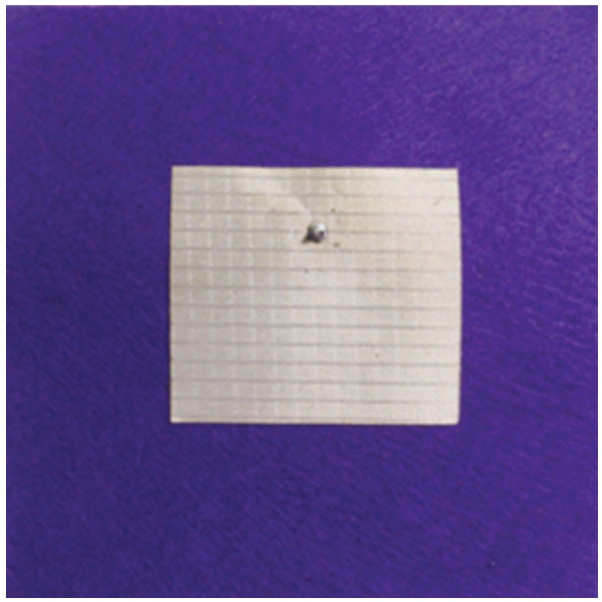

(b)

Figure 7: Patch antenna on denim (a) and felt (b) using Shieldit Super electrotextile.

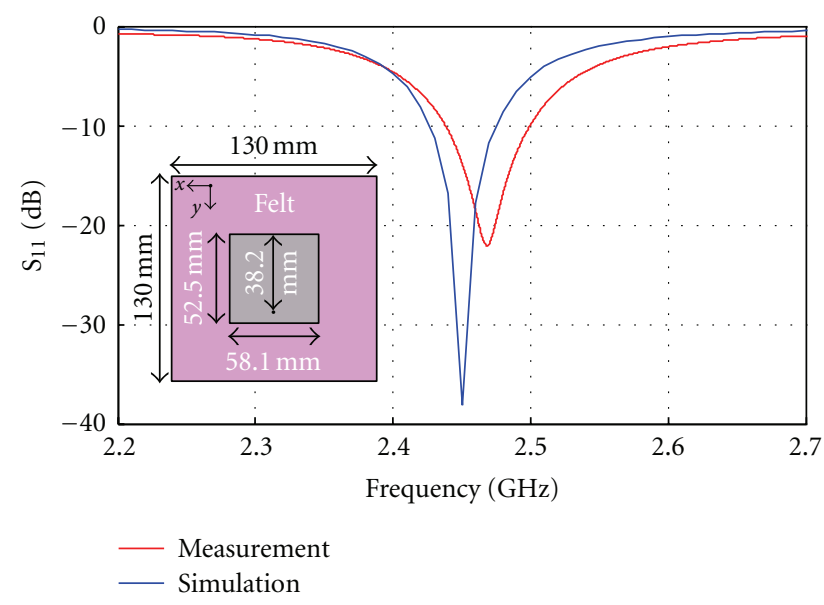

(a)

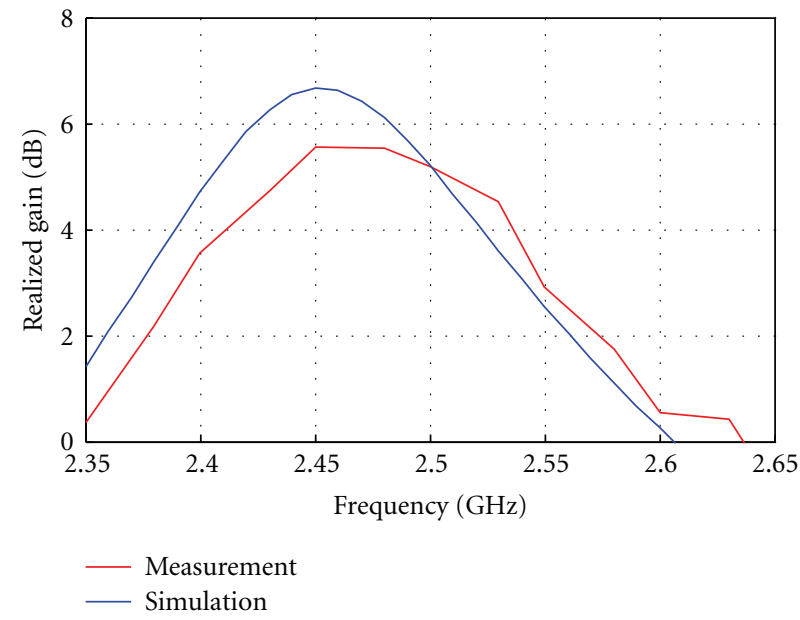

(b)

FIGURE 8: Prototype geometry description and characterization results for patch antenna on felt: return loss (a) and realized gain (b). 
TABLE 1: Properties of electrotextiles.

\begin{tabular}{lcc}
\hline Electrotextile & Pure Copper Taffeta Fabric & Shieldit Super \\
\hline Measured resistance (IETR) & $0.031 \mathrm{Ohm} / \mathrm{sq}$ & $0.025 \mathrm{Ohm} / \mathrm{sq}$ \\
Surface resistance & $0.05 \mathrm{Ohm} / \mathrm{sq}$ & $<0.1 \mathrm{Ohm} / \mathrm{sq}$ \\
Conductivity & $2.5 \times 10^{5} \mathrm{~S} / \mathrm{m}$ & $6.67 \times 10^{5} \mathrm{~S} / \mathrm{m}$ \\
Thread pitch & $0.232 \mathrm{~mm}$ & $0.146 \mathrm{~mm}$ \\
Conductive element & $\mathrm{Cu}$ & $\mathrm{Ni}$ and $\mathrm{Cu}$ \\
Adhesive backing & $\mathrm{No}$ & Yes \\
Thickness & $0.08 \mathrm{~mm}$ & $0.17 \mathrm{~mm}$ \\
Weight & $80 \mathrm{~g} / \mathrm{m}^{2}$ & $230 \mathrm{~g} / \mathrm{m}^{2}$ \\
\hline
\end{tabular}

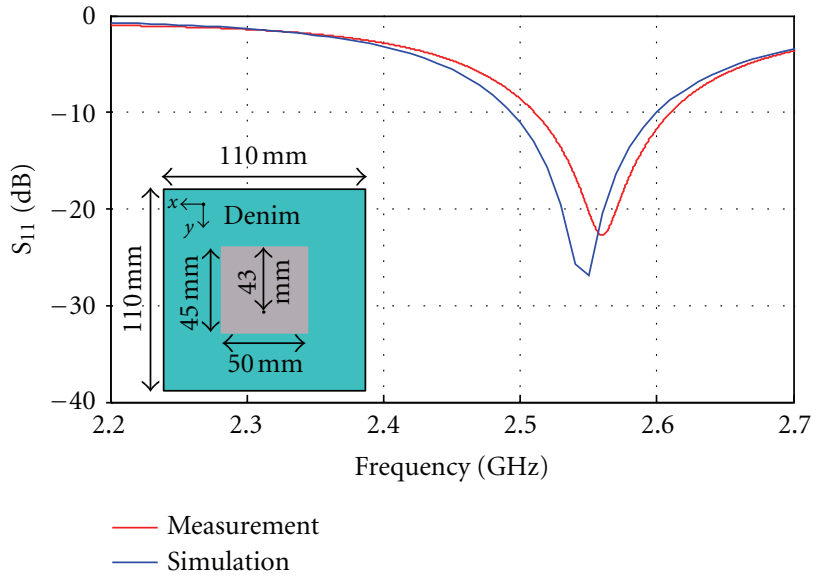

(a)

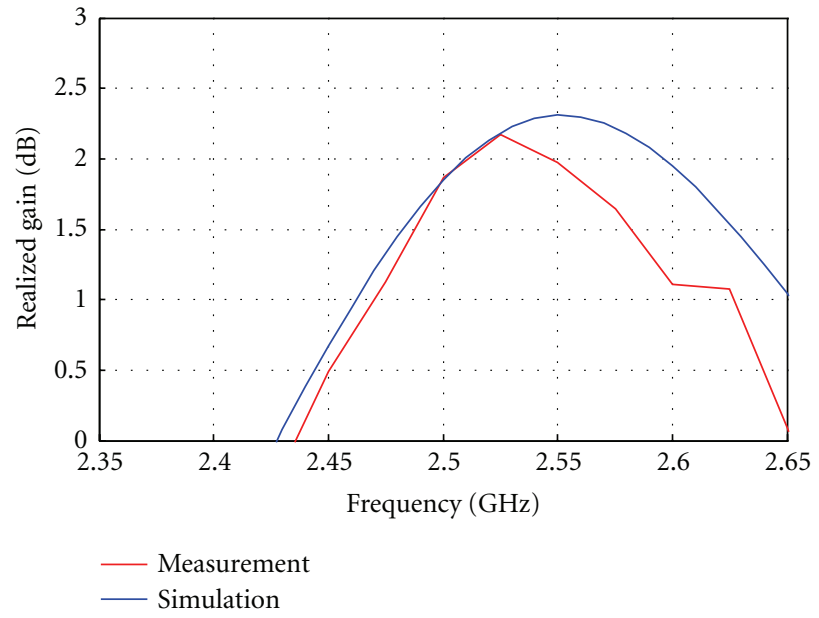

(b)

FIGURE 9: Prototype geometry description and characterization results for patch antenna on denim: return loss (a) and realized gain (b).

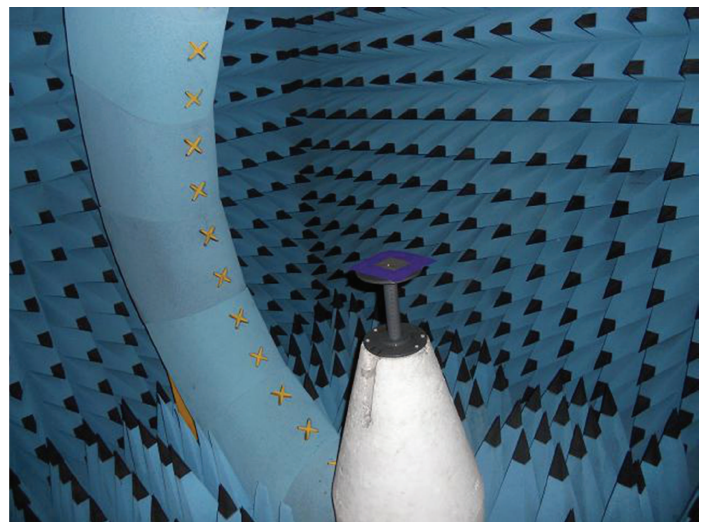

FIGURE 10: Antenna's measurement setup in Satimo stargate SG 32 anechoic chamber.

lower frequency and the upper band bandwidth. To achieve dual-band operation at the intended frequencies, the unitcell dimensions are optimized. The optimized values are given in Table 2.

According to simulation results shown in Figure 13 and detailed on Table 3, the AMC behavior of the structure
TABLE 2: Unit-cell dimensions for dual hexagonal AMC.

\begin{tabular}{lcccc}
\hline \multicolumn{5}{c}{ Dimensions $(\mathrm{mm})$} \\
\hline Hex_out & Hex_cut & Hex_in & $\mathrm{dL}$ & $h$ \\
15.7 & 12 & 11.5 & 0.8 & 4.0 \\
\hline
\end{tabular}

TABLE 3: Frequency bands and bandwidths for dual-band hexagonal AMC.

\begin{tabular}{lccccc}
\hline & \multicolumn{3}{c}{ Frequencies $(\mathrm{GHz})$} & \multicolumn{2}{c}{ Bandwidth } \\
& $f_{\text {Low }}$ & $f_{r}$ & $f_{\mathrm{Up}}$ & Total $(\mathrm{MHz})$ & $\%$ \\
\hline Lower band & 2.40 & 2.55 & 2.70 & 300 & 11.76 \\
Upper band & 5.04 & 5.50 & 6.04 & 1000 & 18.18 \\
\hline
\end{tabular}

takes place from $2.4 \mathrm{GHz}$ to $2.7 \mathrm{GHz}$ and from $5.04 \mathrm{GHz}$ to $6.04 \mathrm{GHz}$ covering all the WiFi bands.

\section{Design of a Novel Broadband Textile Antenna}

To show the capabilities of the AMC reflector announced in the previous section in terms of dual-band operation and backward radiation reduction, a novel CPW-fed broadband antenna is designed to be placed over it. 


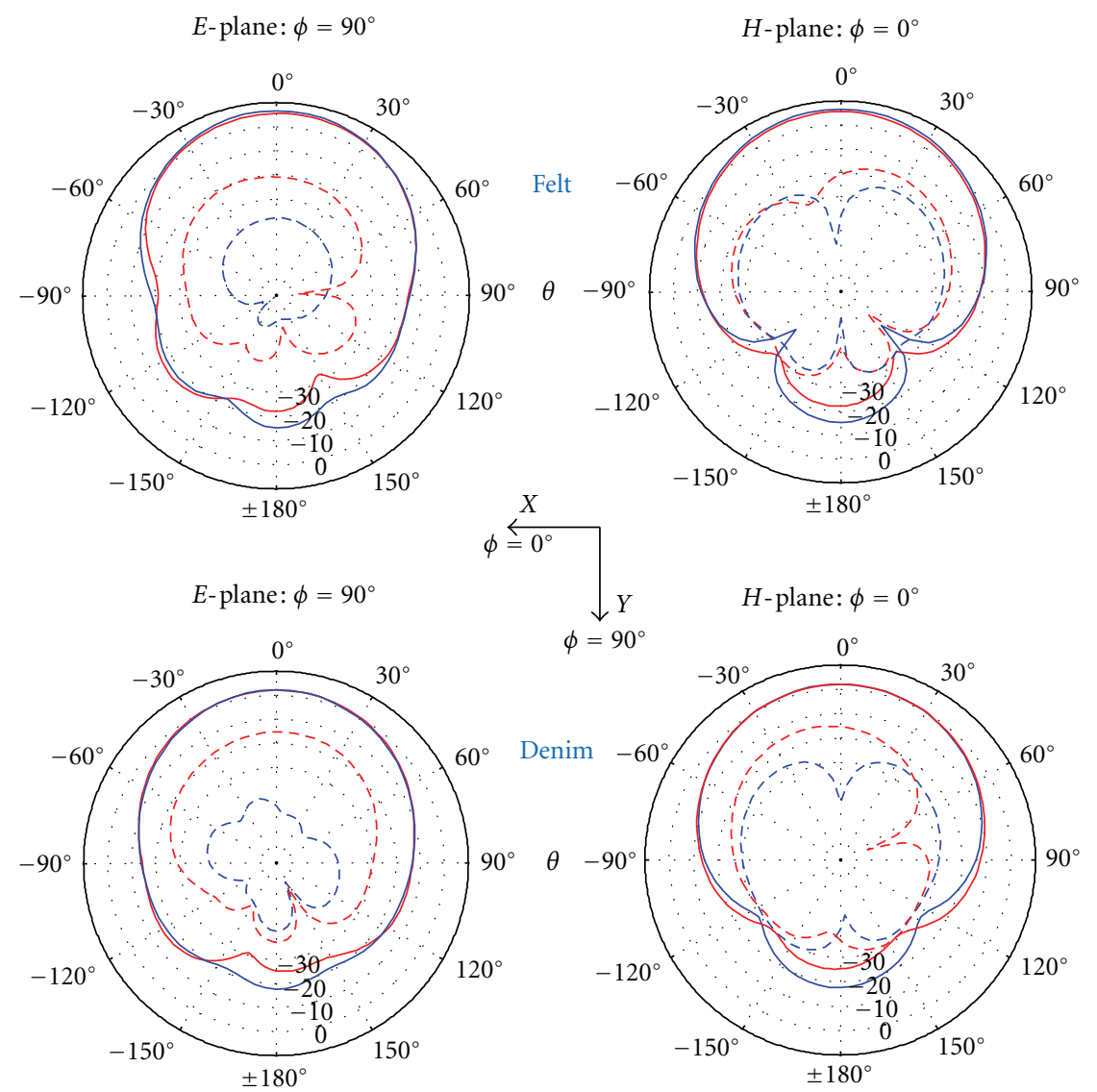

FIGURE 11: Radiation pattern of the patch antennas on felt $(f=2.450 \mathrm{GHz})$ and denim $(f=2.525 \mathrm{GHz})$ substrates. Solid curves present the principal polarization (PP) and the dotted ones the cross polarization (XP). Blue curves correspond to the simulation patterns and red curves to the measured ones.

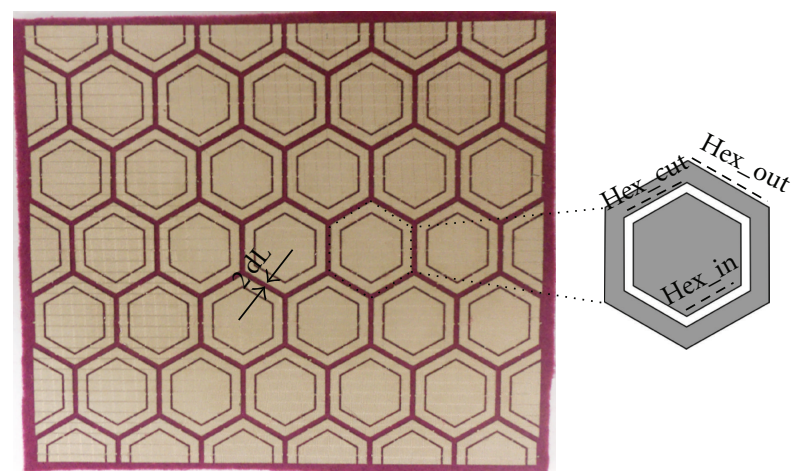

FIgURE 12: Dual-band textile hexagonal AMC-manufactured prototype: top view and unit-cell detail.

The geometry of the proposed CPW-fed broadband antenna is shown in Figure 15. The antenna consists of a rectangular radiating element with two slots and fed by a CPW line. Shieldit Super electrotextile is used to implement these conductive parts which are adhered onto the same side of a $2 \mathrm{~mm}$ thick felt substrate.

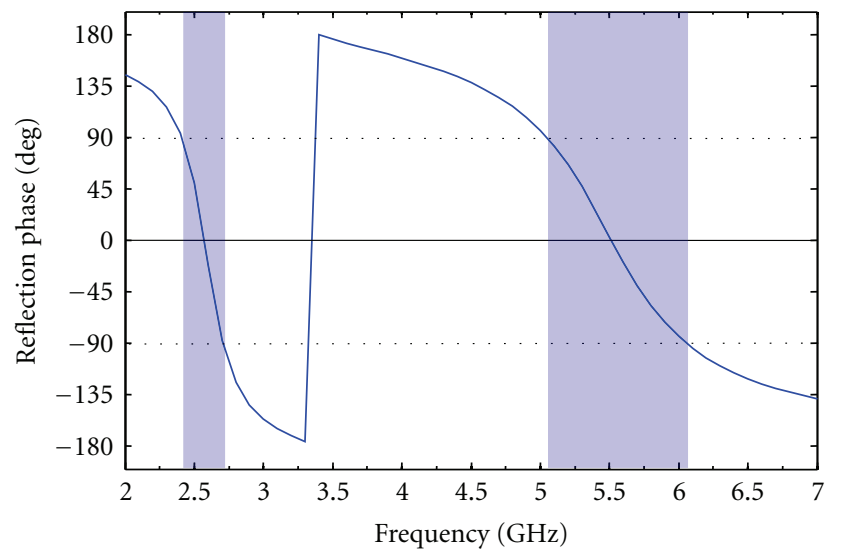

FIGURE 13: Simulated reflection phase of the AMC surface.

The antenna geometry has been optimized to cover the $S_{11}$ bandwidth from $2 \mathrm{GHz}$ to $6 \mathrm{GHz}$. The resulting optimized dimensions are given in Figure 14.

In Figure 15 is presented the measured and simulated return loss of the manufactured CPW-fed broadband antenna. The measurement results yielding a bandwidth 


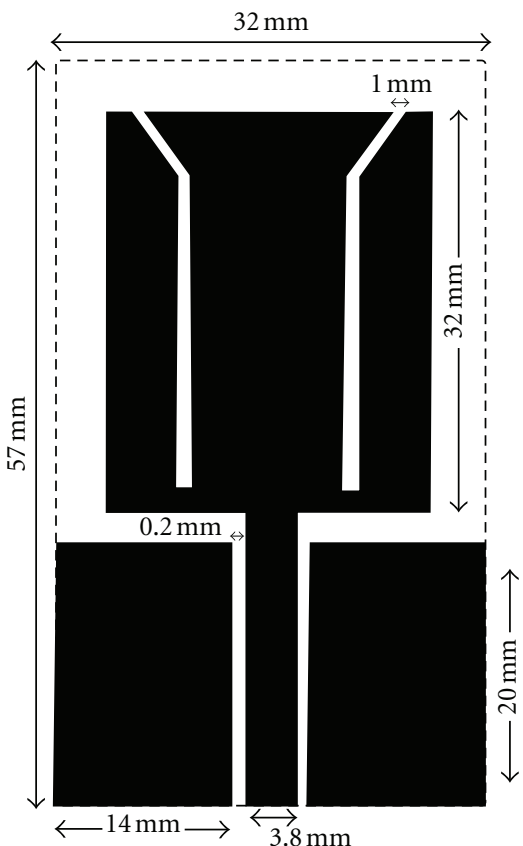

(a)

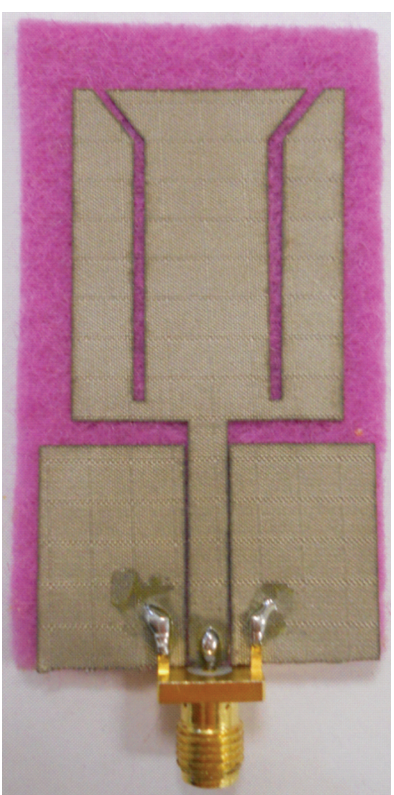

(b)

FIGURE 14: CPW-fed broadband antenna geometry and prototype.

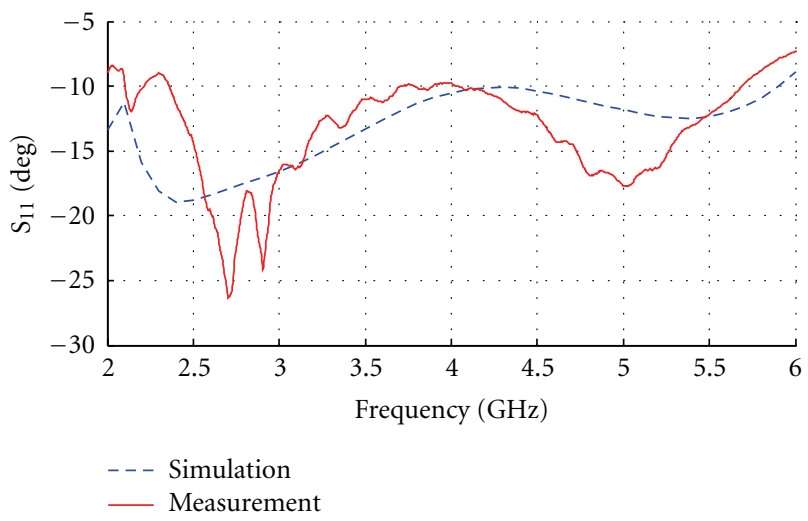

FIGURE 15: Simulated and measured return loss for the CPW-fed broadband monopole antenna on felt.

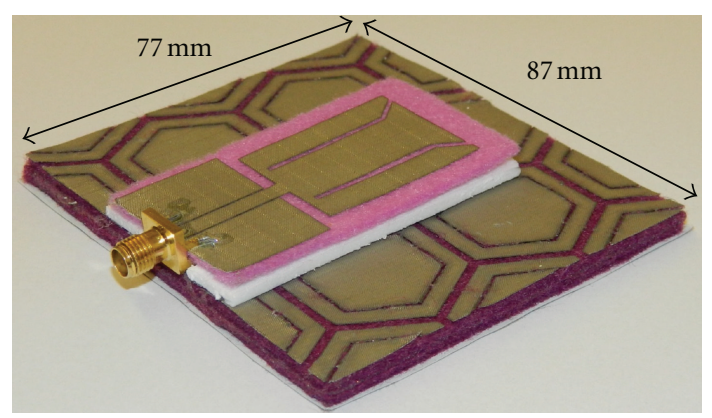

Figure 16: CPW-fed antenna on AMC prototype using felt and electrotextile.

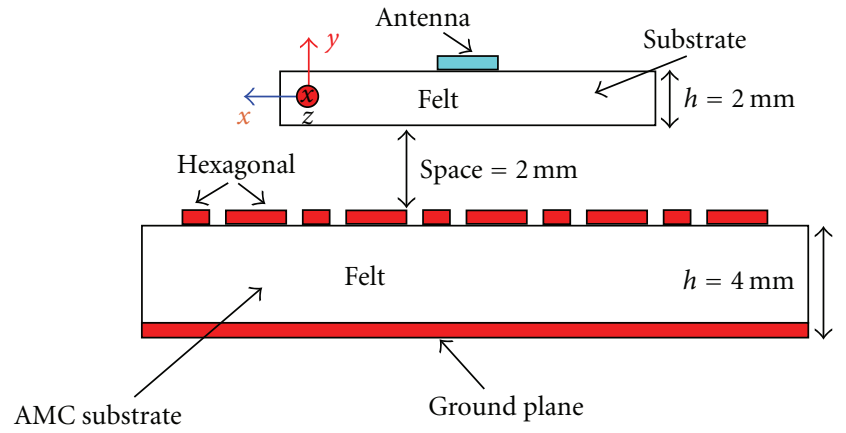

FIGURE 17: Side view of CPW-fed antenna on AMC prototype.

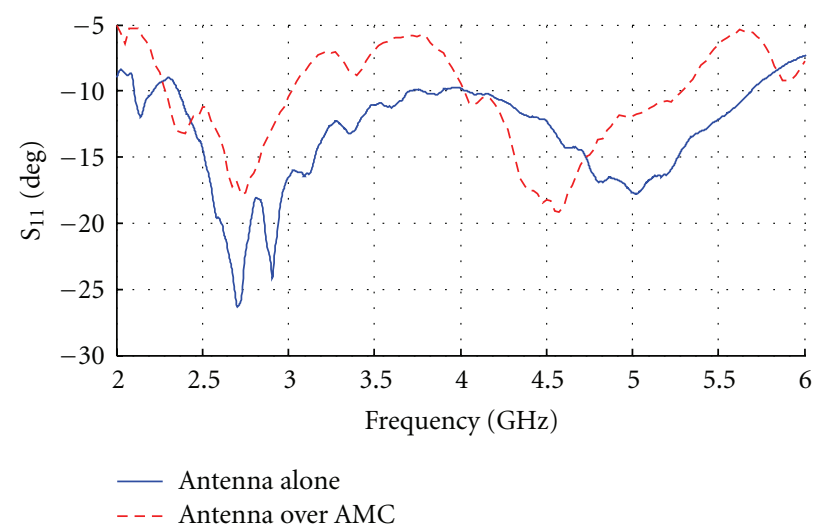

FIGURE 18: Return loss measurement results for the CPW-fed antenna alone and for the CPW-fed antenna on AMC. 

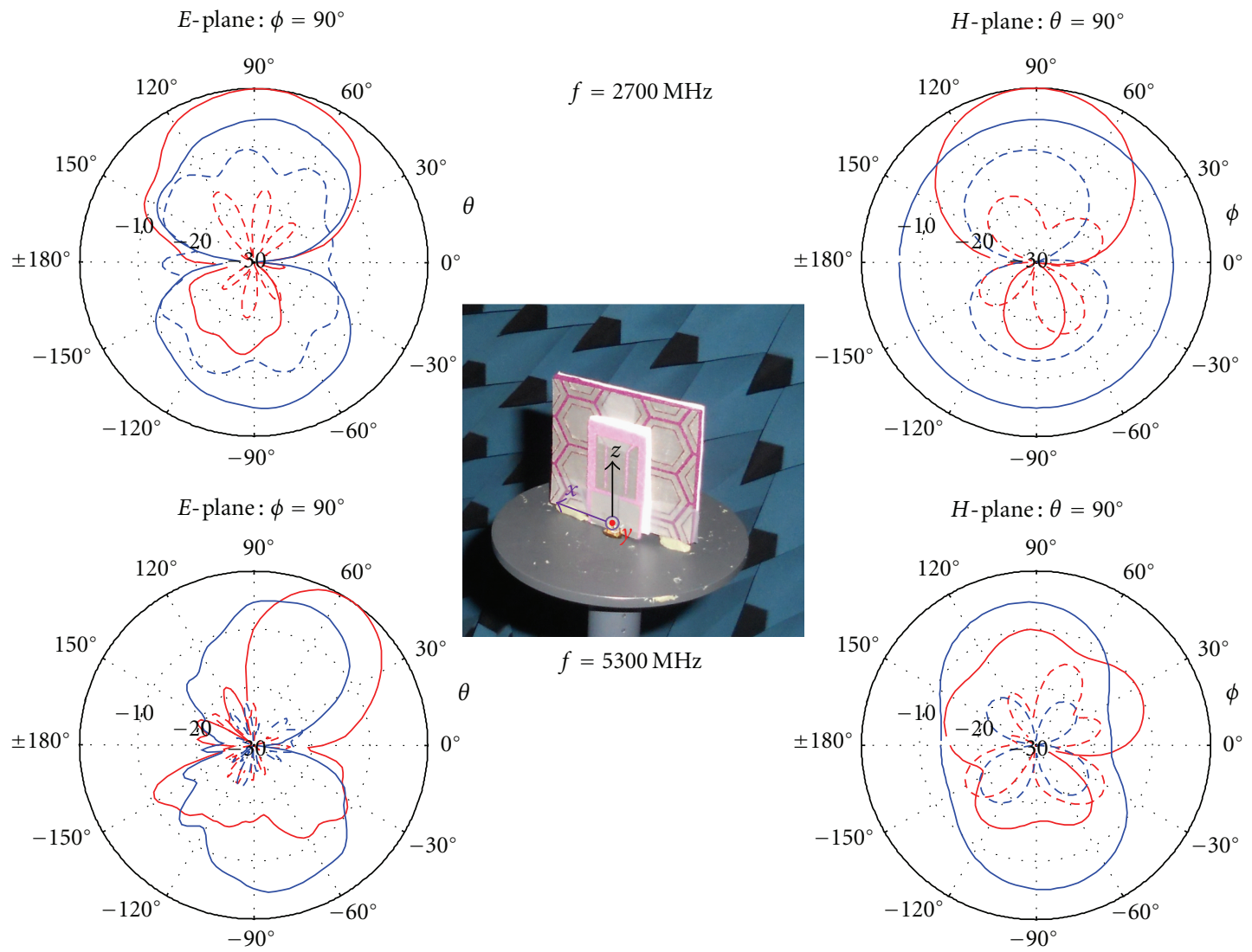

FIGURE 19: Measured radiation patterns (PP in solid line and XP in dashed line) at $f=2.700 \mathrm{GHz}$ and $f=5.300 \mathrm{GHz}, \mathrm{CP}$, for the CPW-fed monopole antenna without (blue) and with (red) AMC reflector, manufactured on felt substrate.

from $2.1 \mathrm{GHz}$ to $5.7 \mathrm{GHz}$ agree quite well with simulation ones exhibiting proper matching from $2 \mathrm{GHz}$ to $5.9 \mathrm{GHz}$. A slight shift in frequency mainly due to manufacturing tolerances can be observed.

$E$-plane and $H$-plane normalized radiation pattern are depicted in Figure 19 at $2.7 \mathrm{GHz}$ and $5.3 \mathrm{GHz}$. As it is expected for a monopole antenna alone (in free space), the $H$-plane pattern is almost omnidirectional. The realized gain versus frequency is shown in Figure 20.

\section{Antenna over AMC on Textile}

Figure 16 shows the manufactured prototype of CPW-fed antenna on AMC using felt and electrotextile. Between the antenna and the AMC, there is a $2 \mathrm{~mm}$ foam layer (see Figure 17). The prototype dimensions are $87 \mathrm{~mm} \times 77 \mathrm{~mm}$.

In Figure 18, the measured return loss for the CPWfed antenna on AMC and for CPW-fed antenna alone is presented for comparison. It can be observed that a dualband performance is obtained for the combined structure due to the AMC dual-band behavior. A slight frequency shift to lower frequencies also appears due to capacitive effects when placing the antenna over the AMC. Finally, the radiation patterns for both prototypes are shown in Figure 19, whereas the gain has been depicted in Figure 20. As it could be expected, the backward radiation of the

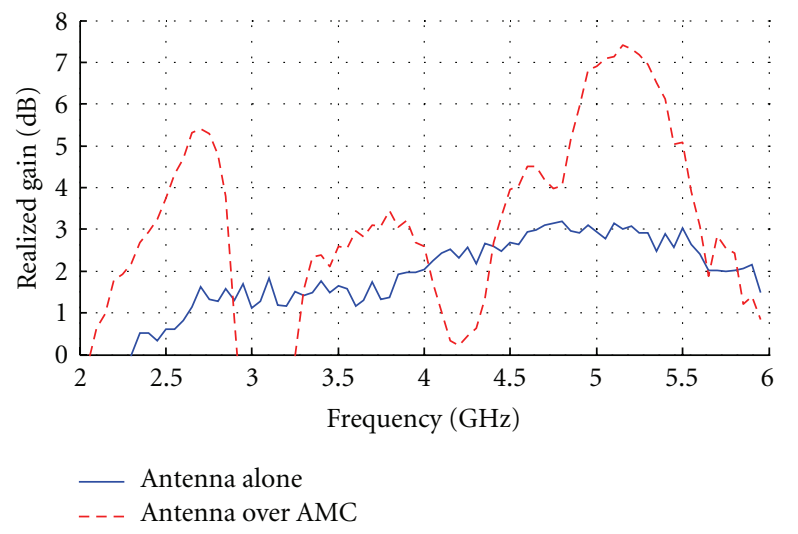

FIGURE 20: Measured realized gain for the CPW-fed monopole antenna alone and for the CPW-fed monopole antenna over AMC.

antenna is reduced by the AMC reflector and the gain is considerably improved.

\section{Conclusions}

Two methods for fabric characterization are presented in this paper: one working at a single frequency and the other on a broadband range. They allowed to retrieve the permittivity 
of felt and denim materials. Based on these data, two patch antennas are realized on these materials. We obtained a very good agreement between the simulation and experimental results for return loss, as well as for the gain parameters, confirming the reliability of the retrieved data.

One of the major contributions of this paper is the design of a textile AMC of original shape. This reflector has been designed to be combined with the textile antennas in order to protect the human body from radiation in one hand and to improve the antenna performance in the other hand. AMC structure has a dual-band behavior and is intended to cover both of the Wifi bands.

To examine the validity of the AMC properties, we introduced a novel CPW-fed broadband antenna (working on the whole frequency range from low Wifi band to high Wifi band), realized it on felt substrate, and placed it over the AMC. A remarkable enhancement is obtained for the backward radiation and the gain of the combined structure compared to those of the CPW-fed antenna alone, concluding to the necessity of AMC reflectors for the onbody applications.

\section{Acknowledgments}

This work has been supported by "Agence Nationale de la Recherche" (ANR), France, under Grants ANR-09-VERS003 for the METAmateriaux pour VESTements intelligents (METAVEST) project and also by the "Images et Réseaux" cluster.

\section{References}

[1] B. Gupta, S. Sankaralingam, and S. Dhar, "Development of wearable and implantable antennas in the last decade: a review," in Proceedings of the 10th Mediterranean Microwave Symposium, (MMS '10), pp. 251-252, August 2010.

[2] L. Vallozzi, W. Vandendriessche, H. Rogier, C. Hertleer, and M. L. Scarpello, "Wearable textile GPS antenna for integration in protective garments," in Proceedings of the 4th European Conference on Antennas and Propagation, (EuCAP '10), April 2010.

[3] I. Locher, M. Klemm, T. Kirstein, and G. Tröster, "Design and characterization of purely textile patch antennas," IEEE Transactions on Advanced Packaging, vol. 29, no. 4, pp. 777788, 2006.

[4] D. Sievenpiper, L. Zhang, R. F. Jimenez Broas, N. G. Alexöpolous, and E. Yablonovitch, "High-impedance electromagnetic surfaces with a forbidden frequency band," IEEE Transactions on Microwave Theory and Techniques, vol. 47, no. 11, pp. 2059-2074, 1999.

[5] F. R. Yang, K. P. Ma, M. Yongxi Qian, and T. Itoh, "A uniplanar compact photonic-bandgap (UC-PBG) structure and its applications for microwave circuits," IEEE Transactions on Microwave Theory and Techniques, vol. 47, no. 8, pp. 1509$1514,1999$.

[6] M. E. De Cos, Y. Álvarez, R. C. Hadarig, and F. LasHeras, "Flexible uniplanar artificial magnetic conductor," in Proceedings of the Electromagnetic Research (PIER '10), vol. 106, pp. 349-362, July 2010.

[7] M. E. De Cos, Y. Álvarez, and F. Las-Heras, "Planar artificial magnetic conductor: design and characterization setup in the RFID SHF band," Journal of Electromagnetic Waves and Applications, vol. 23, no. 11-12, pp. 1467-1478, 2009.

[8] J. Liang and H. Y. D. Yang, "Radiation characteristics of a microstrip patch over an electromagnetic bandgap surface," IEEE Transactions on Antennas and Propagation, vol. 55, no. 6, pp. 1691-1697, 2007.

[9] S. Zhu and R. Langley, "Dual-band wearable textile antenna on an EBG substrate," IEEE Transactions on Antennas and Propagation, vol. 57, no. 4, pp. 926-935, 2009.

[10] M. Mantash, A-C Tarot, S. Collardey, and K. Mahdjoubi, "Dual-band CPW-fed G-antenna using an EBG structure," in Proceedings of the Antennas and Propagation Conference (LAPC '10), pp. 453-456, Loughborough, UK, 2010.

[11] C. Hertleer, A. van Laere, H. Rogier, and L. van Langenhove, "Influence of relative humidity on textile antenna performance," Textile Research Journal, vol. 80, no. 2, pp. 177-183, 2010.

[12] D. Liu, U. Pfeiffer, J. Grzyb, and B. Gaucher, Advanced Millimeter-Wave Technologies: Antennas, Packaging and Circuits, chapter 5, Wiley, 2009.

[13] E. Salahun, P. Quéffélec, M. Le Floc'h, and P. Gelin, "A broadband permeameter for "in situ" measurements of rectangular samples," IEEE Transactions on Magnetics, vol. 37, no. 4 I, pp. 2743-2745, 2001.

[14] Ansoft HFSS, Four Station Square Suite 200, Pittsburgh, Pa, USA.

[15] http://www.univ-brest.fr/lest/caracterisation/pages/pages_ francais/.

[16] A. Tronquo, H. Rogier, C. Hertleer, and L. van Langenhove, "Applying textile materials for the design of antennas for wireless body area networks," in Proceedings of the European Conference on Antennas and Propagation (EuCAP'06), no. ESA SP-626, pp. 1-5, Nice, France, November 2006. 

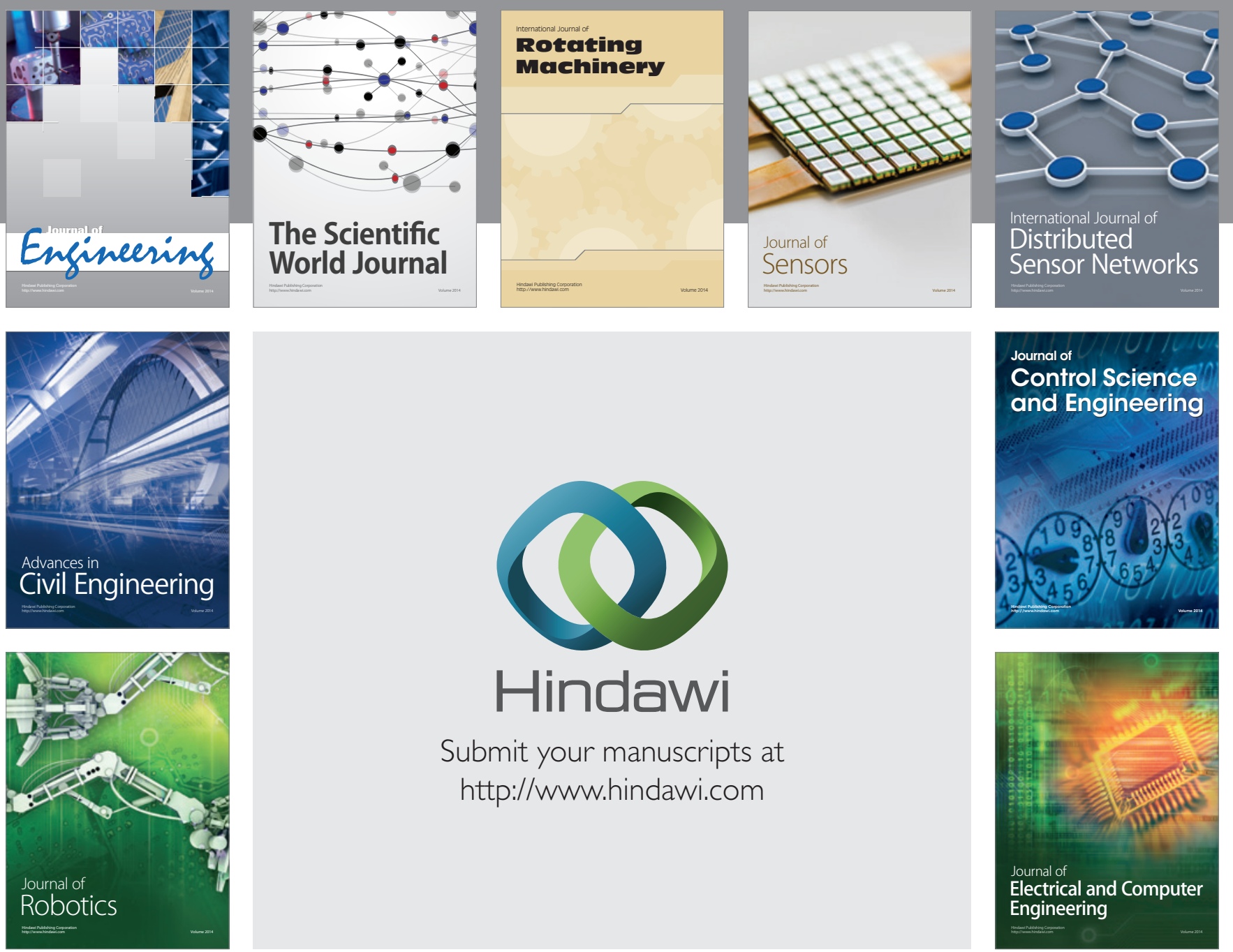

Submit your manuscripts at

http://www.hindawi.com
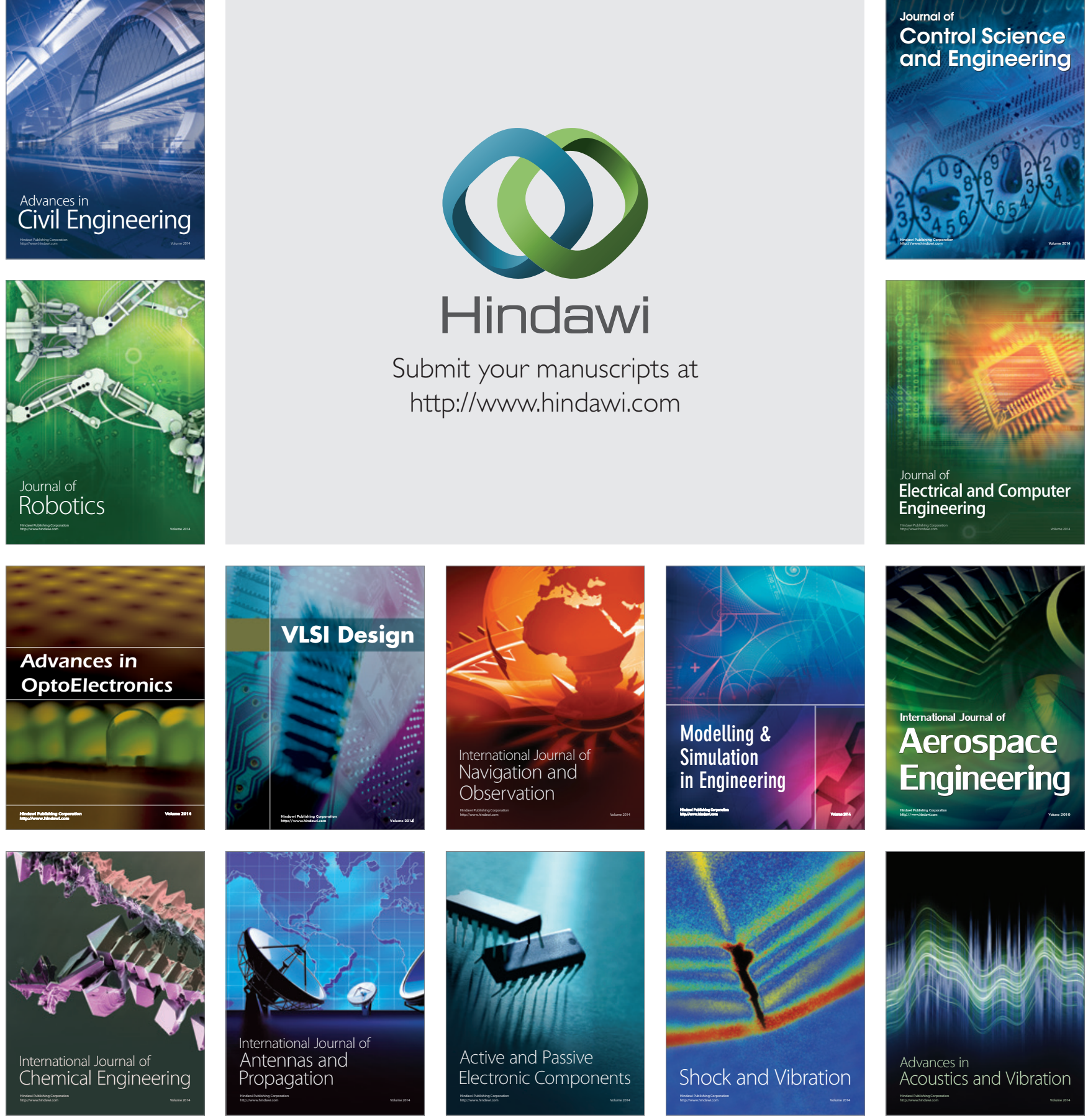